\title{
A Risk Stratification Model for Antihypertensive Medication Non-Adherence Among Chinese Immigrants
}

Asian/Pacific Island Nursing Journal Volume 1(3): 97-104 (C)Author(s) 2016 http://digitalscholarship.unlv.edu/apin/

\author{
Wen-Wen $\mathrm{Li}^{\mathrm{a}}$ and Chih-Ling Huang ${ }^{\mathrm{b}}$
}

\begin{abstract}
The purpose of this study was to establish a risk stratification model for identifying Chinese immigrants at risk for non-adherence to antihypertensive medications. Questionnaires were self-administered to 200 Chinese immigrants in San Francisco, USA. Questionnaires included demographics, culture factors (e.g., Perceived Susceptibility in General, Perceived Benefits of Western Medication, Perceived Benefits of Chinese Herbs, and HealthRelated Social Support), and medications adherence. Participants' mean age was $70.6( \pm 10.3)$ years. Three stratification factors were identified for non-adherence: Lower Perceived Susceptibility in General, lower Perceived Benefit of Western Medications, and longer Length of Stay in the United States. The probability of non-adherence was $77 \%, 62 \%$, and $57 \%$ for lower perceived susceptibility, longer stay in the United States, and lower perceived benefits of Western medications, respectively. A combination of lower perceived susceptibility and lower perceived benefits of medication predicted $81 \%$ non-adherence and lower perceived susceptibility with longer stay in the United States predicted at $84 \%$. All three factors combined predicted nearly $90 \%$. Patients with all three factors had the highest risk for non-adherence. The second priority groups are patients with lower perceived susceptibility and those with lower perceived susceptibility combined with any of the other two factors. In the clinical setting, these three groups are a high priority for education on the importance of medication adherence.
\end{abstract}

Keywords: risk stratification, risk behavior, chronic illness, cultural sensitivity, medication adherence

Hypertension (HTN) is a common medical condition that plagues about 1 billion of the world's population (World Health Organization, 2016). In developed countries, uncontrolled HTN increases the risk of stroke, coronary artery disease, and renal failure (World Health Organization, 2016). HTN can be treated with a variety of anti-hypertensive medications that control blood pressure; however, failure to adhere to the treatment regimen can be a problem for many patients in part because HTN does not present with symptoms (Chockalingam, 2008; Joint National Committee, 2003). Medication adherence requires the patient to follow the prescription at least $80 \%$ of the time to ensure effectiveness (Ingersoll \& Cohen, 2008). Low medication adherence (less than $80 \%$ ) in patients with HTN can greatly impede the progress of blood pressure control (Joint National Committee, 2003). The cost for taking care of patients with nonadherence exceeds that of adherent patients (Dragomir et al., 2010). Thus, development of a risk stratification model to identify patients who are at risk for antihypertensive medication non-adherence is important to help clinicians plan a more effective HTN management program for the target population.

Chinese immigrants constitute the largest proportion $(22 \%)$ of Asian Americans and Pacific Islanders (17.3 million in the year 2010) in the United States (U.S. Census Bureau., 2012). More than 2 million Chinese reside in the United States (U.S. Census Bureau, 2013); of this group, roughly $40 \%$ have been diagnosed with HTN (Chen \& Hu, 2014; Sheng et al., 2013), compared to $29 \%$ in the general population

${ }^{a}$ San Francisco State University, San Francisco, CA, USA

${ }^{b}$ Chang Jung Christian University, Tainan, Taiwan

Corresponding Author:

Wen-Wen Li, San Francisco State University

School of Nursing

Email: wenwenli@sfsu.edu 
(Egan, Zhao, \& Axon, 2010). Chinese immigrants have their own cultural background and traditional medical practices, which can complicate medication treatment for HTN (Taiwan Government Information Office, 2015). Therefore, developing a risk stratification model using predictors, such as cultural health perceptions of HTN, its treatment, and length of stay (LOS) in the United States, should be taken into consideration when helping Chinese immigrant patients manage their HTN (Li, Stewart, Stotts, \& Froelicher, 2005; Li, Stotts, \& Froelicher, 2007). Both cultural health perceptions of HTN and LOS in the United States were associated with acculturation so they were used as cultural predictors (Abraído-Lanza, Armbrister, Flórez, \& Aguirre, 2006; Li, Wallhagen, \& Froelicher, 2008). Identifying at-risk Chinese immigrants for poor medication adherence using a risk stratification model and working with them to improve their HTN management is critical for preventing serious HTN-related complications, such as stroke. This in turn helps achieve a national goal of reducing health disparities.

Another strength of a risk stratification model is to provide a predictive number on the probability that patients will be non-adherent if they have certain risk factors (Long \& Freese, 2006). A concrete number on the probability of medication non-adherence can give clinicians the confidence to more accurately identify priority patients they should work closely with to improve their HTN management and use health care resources wisely. In addition, the probability of nonadherence can serve as an outcome measure in conjunction with blood pressure measures to help clinicians monitor patient progress in HTN management.

Given the above, the specific aims of this study were to challenge the traditional approach of simply presenting an odds ratio of a single predictor to provide a better risk stratification model by (a) using combined, culturally specific, risk stratification factors for identifying Chinese immigrants at risk for non-adherence to antihypertensive medications; and (b) predicting a probability of non-adherence for patients with a certain subset of risk stratification factors. Currently, these approaches are rarely used in health care research because of a lack of appropriate statistical software to generate a risk stratification model. However, a relatively new statistical program (SPost in Stata) has been developed in the past decade, which will generate such a stratification model to predict the probability of non-adherence (Long \& Freese, 2006). Due to a good match between this software and the specific aims of this study, SPost in Stata was used for data analysis.

Findings from this study may advance health care by establishing a culturally specific, risk stratification model to better identify Chinese immigrants who are at risk for non-adherence to antihypertensive medication. In addition, this risk stratification model may also be used to help identify other Asian immigrants at risk for non-adherence, such as Vietnamese, who share a similar cultural background with Chinese. To our knowledge, this is the first study of this type in this population.

\section{Methods}

\section{Study Design}

The parent study with a longitudinal design was completed in 2007. The data presented here are a secondary analysis based on a cross-sectional survey (Li, Stewart, Stotts, \& Froelicher, 2006) conducted during the first interview asking about demographic variables, cultural factors, and medication adherence.

\section{Sample and Settings}

A convenience sample of 200 Chinese immigrants was recruited from a nonprofit Asian health clinic in the San Francisco Bay Area. This sample size was determined by using nQuery Advisor (Elashoff, 2007). When the sample size is 200 , the logistic regression test of $\alpha=.05$ (two-sided) will have more than $80 \%$ power to detect an odds ratio of 2.5 .

The inclusion criteria were (a) Chinese immigrant (ethnic Chinese, born outside of the United States), (b) aged 18 years or older, (c) diagnosis of HTN, (d) on antihypertensive medication for more than one month prior to the study, and (e) able to speak and read Chinese. The exclusion criteria were medical instability (e.g., end-stage renal failure) and psychiatric problems (e.g., schizophrenia), which can compromise study participation.

\section{Data Collection Procedure}

Institutional Review Board approval was obtained from the University of California, San Francisco and written consents were obtained from all participants. Subjects with HTN were identified through a computerized database. An invitation letter in Chinese that described the study purpose was sent to all potentially eligible participants. A postcard to decline participation was also included. Calls were made to eligible participants who accepted the invitation through the mail to discuss further interest in participating in the study. Participants were asked to bring all their medications with them to their next clinical visit where their blood pressure will be measured. Questionnaires were self-administered and completed by the participants. 


\section{Measurement of Risk Stratification Varia- bles}

Seven potential risk stratification, cultural factors were measured via self-administered questionnaires. The detailed description and scoring methods have been published elsewhere (Li et al., 2005). Herein, we will briefly report definitions for each scale. The psychometric properties are listed in Table 1 (Li et al., 2005).

Perceived Susceptibility in General (PSG) is the patient's perception of how susceptible she or he is to the impact of HTN. This impact includes a decline in the quality of life due to disease. For example, "If I had a stroke or heart disease, it is possible that I will need others to help for the rest of my life." Perceived Susceptibility to Specific Diseases (PSSD) is the patient's perception of how susceptible she or he is to the effects of HTN (e.g., experiencing complications due to uncontrolled HTN [i.e., heart attack, stroke, and kidney disease]). Perceived Benefits of Chinese Herbs in General (PBCHG) is an individual's perception of benefits of using Chinese herbs, such as fewer side effects compared to Western medications. Perceived Benefits of Western Medications (PBWM) for Hypertension evaluates how participants viewed the effectiveness of Western medications in lowering blood pressure. Health-Related Social Support (HRSS) is an individual's perception of support provided by family and/or friends for health-related activities (e.g., having someone available to discuss health issues before going to the doctor). Social Support in General (SSG) is defined as perceived tangible and emotional support from family or friends (e.g., having someone available whom an individual can count on to listen to her when she needs to talk). Length of Stay (LOS) in the United States asks how long has an individual lived in the United States measured in years.

Table 1. Psychometric Properties for Culturally Specific Risk Stratification Factor Scales

\begin{tabular}{lccc}
\hline \multicolumn{1}{c}{ Scales (number of items) } & Possible range & Mean $(S D)$ & Cronbach's $\alpha$ \\
\hline Perceived Susceptibility in General (2) & $2-10$ & $7.99(1.21)$ & 0.66 \\
Perceived Susceptibility to Specific Diseases (5) & $5-25$ & $10.25(4.04)$ & 0.79 \\
Perceived Benefits of Western Medications for Hypertension (2) & $2-10$ & $7.95(1.01)$ & 0.57 \\
Perceived Benefits of Chinese Herbs in General (4) & $4-20$ & $14.01(2.22)$ & 0.75 \\
Health-Related Social Support (7) & $7-35$ & $26.85(3.77)$ & 0.83 \\
Social Support in General (6) & $6-30$ & $19.18(6.60)$ & 0.91 \\
\hline
\end{tabular}

Note. The higher the score, the higher the level of perception or social support (e.g., greater susceptibility; Li et al., 2006).

\section{Measurement of Demographic Variables}

The demographic data including age, gender, education, religion, annual family income, born country, and living with family members, were measured using a self-report questionnaire. The questionnaire was modified from the Behavioral Risk Factor Surveillance System Survey Questionnaire (Centers for Disease Control and Prevention, 1998).

\section{Measurement of Outcome Variables}

The Medication Adherence Scale (Morisky, Green, \& Levine, 1986) evaluating the frequency of forgotten or missed medications, and how often they were careful to take their antihypertensive medications was slightly altered (Li et al., 2005). A Likert scale with 5 response choices (ranging from "None of the time" to "All of the time") was used to replace the original dichotomous one due to response preference indicated by Chinese immigrant participants in our pilot study ( $\mathrm{Li}$ et al., 2005). Based on the pilot study findings, the Medication Adherence Scale was found to be superior to other measures of adherence, such as pill counts and medication events monitoring systems for Chinese immigrant patients ( $\mathrm{Li}$ et al., 2005; Li et al., 2006; Morisky et al., 1986). Thus, it was used in this study for measuring medication adherence. Medication adherence was defined as having at least $80 \%$ of the highest score on the Medication Adherence Scale (Ingersoll \& Cohen, 2008). Thus, $80 \%$ was used as a cut point for dichotomizing this outcome variable into adherence and non-adherence.

Blood pressure was measured twice based on the standard procedures provided by the Joint National Committee (2003). All participants' blood pressures were measured by the same device and same data collector. Due to the limitation that not all participants could be scheduled for a similar time window (such as early morning) to obtain the most comparable blood pressure outcomes, participants' blood pressure was measured twice and averaged (Joint National Committee, 2003). Blood pressure control was defined as a blood pressure of less than $140 / 90 \mathrm{mmHg}$. For this analysis, blood pressure outcome was only used to describe the sample as it was not a major outcome for the prediction model. 


\section{Data Analysis}

To establish a risk stratification model and the prediction of probability of medication nonadherence, SPost in Stata (version 11.0) was used for data analysis. In order to use SPost, a multiple logistic regression presented in the form of odds ratio with a 95\% CI (Afifi \& Clark, 1997) was performed initially to identify risk stratification factors for medication non-adherence. Logistic regression analysis was used for the analysis because it does not require the assumption of normality of data and it is easy to interpret study results in the form of odds ratio with a 95\% CI (Afifi \& Clark, 1997). Many risk factors were identified from the literature to establish a more robust, stable risk stratification model, and they were included in the regression model, covering (a) demographic factors, such as education, religion, annual family income, born country, and living with family members; and (b) cultural factors, such as perceived benefits of using Chinese herbs versus of using Western medications and LOS in the United States. Since the literature suggested that most of the demographic variables would not be predictors for medication non-adherence (Li, Kuo, Hwang, \& Hsu, 2012; Li, Wallhagen, et al., 2008), they were controlled for in the prediction model instead of being used as independent variables. The median was used as a cut point for defining dichotomous independent variables for a logistic regression analysis for nonadherence. The reason that the independent variables were dichotomized was for ease of interpretation and establishment of a risk stratification model.

Table 2. Multivariate Logistic Regression of Risk Stratification Factors for non-Adherence

\begin{tabular}{|c|c|c|c|}
\hline \multirow[b]{2}{*}{ Variables } & \multirow[b]{2}{*}{$A O R$} & \multicolumn{2}{|c|}{$95 \% \mathrm{CI}$} \\
\hline & & Upper & Lower \\
\hline \multicolumn{4}{|c|}{ Perceived Susceptibility in General* } \\
\hline$>8$ & 1 & & \\
\hline$\leq 8$ & 4.57 & 1.79 & 11.71 \\
\hline \multicolumn{4}{|c|}{ Perceived Susceptibility to Specific Diseases } \\
\hline$>10$ & 1 & & \\
\hline$\leq 10$ & 1.80 & 0.87 & 3.74 \\
\hline \multicolumn{4}{|c|}{ Perceived Benefits of Western Medications for Hypertension* } \\
\hline$>7$ & 1 & & \\
\hline$\leq 7$ & 3.16 & 1.30 & 7.70 \\
\hline \multicolumn{4}{|c|}{ Perceived Benefits of Chinese Herbs in General } \\
\hline$\leq 14$ & 1 & & \\
\hline$>14$ & 0.68 & 0.34 & 1.38 \\
\hline \multicolumn{4}{|c|}{ Health-Related Social Support } \\
\hline$>28$ & 1 & & \\
\hline$\leq 28$ & 1.20 & 0.42 & 3.41 \\
\hline \multicolumn{4}{|c|}{ Social Support in General } \\
\hline$>19$ & 1 & & \\
\hline$\leq 19$ & 1.07 & 0.53 & 2.15 \\
\hline \multicolumn{4}{|c|}{ LOS in the United States* } \\
\hline$\leq 12$ & 1 & & \\
\hline$>12$ & 2.49 & 1.19 & 5.19 \\
\hline \multicolumn{4}{|c|}{ Age (years) } \\
\hline$\leq 72$ & 1 & & \\
\hline$>72$ & 1.01 & 0.48 & 2.11 \\
\hline \multicolumn{4}{|l|}{ Gender } \\
\hline Female & 1 & & \\
\hline Men & 1.16 & 0.60 & 2.25 \\
\hline
\end{tabular}

Note. In the regression model, the controlled variables: Education, religion, annual family income, born country, living with family members; $* x<.05 ; \mathrm{AOR}=$ adjusted odds ratio.

\section{Results}

\section{Sample Characteristics}

The response rate in our study was $80 \%$ (originally, 250 were contacted but 200 actually participated in the study). A detailed description regarding the number of potential participants who were approached, who declined, and who participated was reported previously ( $\mathrm{Li}$ et al., 2006). The mean age was $70.6( \pm 10.3)$ ranging from 39 to 91 years. Half of the participants were women $(50 \%)$. Three-quarters were retired (75\%), married (70\%), and lived with an annual household income less than $\$ 19,999$ (71\%). The mean 
LOS in the United States was $13( \pm 7.2)$ years. The majority $(98 \%)$ preferred to communicate with their care provider in Chinese. Half (51\%) had their HTN controlled and $75 \%$ had medication adherence. More detailed descriptions of the sample characteristics were reported previously ( $\mathrm{Li}$ et al., 2006).

\section{Risk Stratification Variables and Medica- tion non-Adherence}

Of the nine independent variables (seven cultural and two demographic factors), there were three identified risk stratification factors that were statistically associated with non-adherence (Table 2). These were low PSG $(O R=4.57,95 \%$ CI $[1.79,11.71])$; low $\operatorname{PBWM}(O R=3.16,95 \% \mathrm{CI}[1.30,7.70])$; and longer LOS in the United States $(O R=2.49,95 \%$ CI [1.19, 5.19]). There were no significant associations between demographic variables and medication non-adherence.

\section{Risk Stratification Model for Predicting Medi- cation non-Adherence}

Based on those three significant factors, we further used SPost to determine a probability of nonadherence. The probability of non-adherence was $77.3 \%, 61.8 \%$ and $57.3 \%$ for lower PSG, longer LOS in the United States and lower PBWM, respectively (Figure 1). A combination of lower PSG and lower PBWM predicted $81.0 \%$ of non-adherence and lower PSG with longer LOS predicted $83.7 \%$ of non-adherence (Figure 2). All three factors combined predicted $86.5 \%$ of non-adherence.

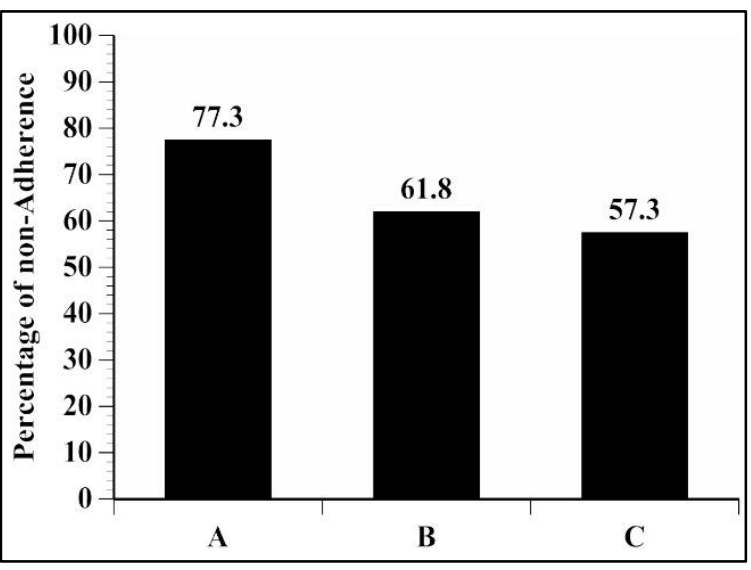

Figure 1. Probability of Medication non-Adherence (Single Factor). A = Low PSG; B = Longer LOS; $\mathrm{C}=$ Low PBWM. Note. PSG $=$ Perceived Susceptibility in General; LOS = Length of Stay in the USA; PBWM = Perceived Benefits of Western Medications.

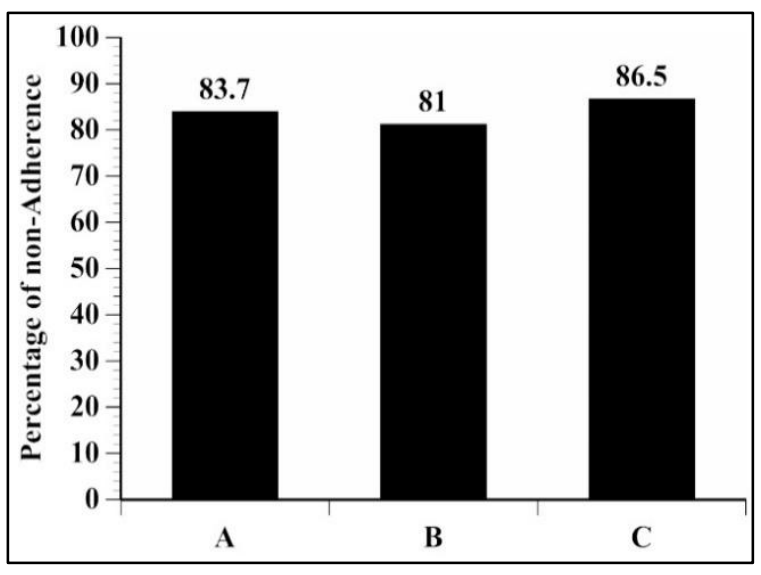

Figure 2. Probability of Medication non-Adherence (Multiple Factors). A = Low PSG + Longer LOS; B = Low PSG + Low PBWM; C = Low PSG + Longer LOS + Low PBWM. Note. PSG = Perceived Susceptibility in General; LOS = Length of Stay in the USA; PBWM = Perceived Benefits of Western Medications.

\section{Discussion}

In brief, the studied population was older and did not speak English, had low levels of education, and poor financial support. Based on the report of a national, multi-center study of atherosclerosis (MESA) study, about one-third of medicated Chinese Americans aged 45-85 years old had uncontrolled HTN (33\%; Kramer et al., 2004). Compared to this population, our sample with a similar age range had a much higher uncontrolled hypertension rate $(49 \%)$. The reason for this may be due to the fact that, compared to MESA participants, our Chinese immigrant participants were less acculturated. For example, nearly $100 \%$ of the participants in this study did not speak English and most stayed within their Chinese community for all activities, such as grocery shopping and attendance at social events. Given this, Chinese immigrants, who share similar living and cultural backgrounds with our sample, such as those who live in Chinatown, warrant further attention of clinical nurses to provide culturally and linguistically appropriate health education to help this population achieve better HTN management.

Among three of the stratification factors, lower PSG was the first and strongest compared to the other two (longer LOS in the United States and lower PBWM); PSG predicted high probability (77\%), of non-adherence in Chinese immigrants. The rationale may be due to the fact that there are no symptoms associated with HTN and many Chinese immigrants may have the perception that there is no need to take their medication, leading to a high level of medication nonadherence. 
In addition, Chinese immigrants have a collectivistic cultural background in which maintaining social order and functioning of the group or family takes precedence (Chen \& Swartzman, 2001; Chen, Tsai, \& Lee, 2009). We found that if an individual had less perceived susceptibility related to HTN (e.g., if a patient is not adherent to antihypertensive medications and had a stroke as a result of non-adherence, family members will need to provide care which may cause compromised relationships in the family), their medication non-adherence was worse. Previously, studies in a Taiwanese population and Chinese immigrants in the United States revealed that the $87 \%$ to $94 \%$ of participants lived with their family, and many of them were family caregivers, 65 years of age and older, and helped care for their grandchildren (Hsu, Mao, \& Wey, 2010; Li et al., 2012). This further illustrates how a patient's social connectivity and responsibility may influence adherence to medication. The strong negative impact on an individual's perception that the health condition will become worse and have an effect on the family, may lead to being greater motivation for better medication adherence.

Longer LOS in the United States also predicted a high probability (62\%) of non-adherence. One explanation might be that the longer Chinese immigrants are in the United States, the more cynical they become toward the Western health system and thus they disregard prescriptions and are reluctant to communicate with their health care providers. For example, if they suffered side effects from a prescribed medication, they may stop taking that medication without consulting their care provider first. Previous research found greater communication barriers between older Chinese (particularly when they stay longer in the United States) and their health care providers than the younger generation, and this barrier was associated with greater medication non-adherence (Turner, Hollenbeak, Weiner, Ten Have, \& Roberts, 2009). Thus, older Chinese immigrants warrant further studies to confirm their greater barriers in being adherent to antihypertensive medications. Although in the literature, LOS is considered one of the factors for acculturation in other immigrant populations (e.g., Filipinos; Abraído-Lanza et al., 2006). For Chinese immigrants, the majority of those who do not speak English and stay in their own communities, LOS in the United States may not be the best indicator for acculturation. Therefore, it is not surprising that longer LOS in the United States is not associated with better adherence. Instead, because of their cynical views toward the Western health care system and maintaining traditional cultural practices, Chinese immigrants with longer LOS in the United States may be more reluctant to be adherent to antihypertensive medications. This warrants further study.
PBWM also predicted a high probability $(57 \%)$ of non-adherence. Although the predicted probability was not as strong as the other two factors, it was suggested by this study and the literature (Li et al., 2005; Li et al., 2012) that PBWM plays a significant role in promoting Chinese immigrants' adherence to antihypertensive medications. Thus, it is essential that nurses should take into consideration when planning health education for Chinese immigrant patients about the management of HTN, which should emphasize the importance of taking Western antihypertensive medications for optimal blood pressure control.

Compared to those studies with only one single risk factor, our results showed that patients with more than one stratification factor had a higher risk for non-adherence. For example, patients with lower PSG (one factor), compared to the other two factors (longer LOS or lower PBWM), had the highest risk of nonadherence $(77 \%)$. However, when lower PSG was combined with longer LOS or lower PBWM, the probability was even higher, ranging from $81 \%-84 \%$. When all three factors were combined, the probability of non-adherence reached nearly $90 \%$.

The above information can help establish a culturally specific, risk stratification model to classify hypertensive patients into different risk groups and direct the focus of clinicians in targeting patients who are at high risk for non-adherence when trying to provide health education regarding HTN management. For example, for patients with all three risk factors, health clinicians should work with family members and other health care team members to develop an optimum intervention, including more frequent followup, culturally and linguistically appropriate health educational programs, and the use of technology, such as social media, to facilitate interaction between patients and care providers. If time and budgets allow, the second priority should be patients with lower PSG and those with lower PSG combined with any of the other two predictors (longer LOS or lower PBWM).

The above risk stratification model warrants further investigation to establish a more robust algorithm to prioritize HTN care for Chinese or those who share similar cultural backgrounds. With the limited budgetary plans in most health care settings, development of such a risk stratification model is important to help clinicians focus care on the patients who are most non-adherent to their antihypertensive medications.

This study has some limitations. One, the Chinese immigrants were recruited from a single region of the San Francisco Bay area. The abundance of Chinese-speaking people in this area and the influence of the Chinese culture on clinical practice could be greater than other regions of the United States. Therefore, results should be carefully assessed in other communities that have fewer Chinese immigrants. For example, Chinese immigrants who live in the Midwest may be moderately acculturated and thus the family 
structure and health beliefs may not be the same. In order to assess the validity of these findings, further validation in other regions is recommended. Second, because of the limitations in our recruitment, study findings may not be generalized to those who are younger, have no language barriers, have higher levels of education, higher incomes, and took antihypertensive medications for less than one month. Moreover, the cross-sectional study design precludes definitive causal associations. Lastly, a larger sample may provide more stable results.

\section{Conclusions}

It is anticipated that these findings can help advance knowledge to improve primary care and education to the most at-risk groups of Chinese immigrants with HTN who are likely to be non-adherent to their medications. Based on our results, we have identified three culturally specific, risk stratification factors that have predicted the probability of medication non-adherence. Of the three factors, lower PSG estimated the highest probability of non-adherence. It is suggested that patients with all three combined factors and patients with lower PSG or lower PSG combined with any of the other two factors (longer LOS in the United States or lower PBWM) warrant special attention by clinicians. Because medication non-adherence continues to be a predicament that prevents patients from achieving good blood pressure control, especially in minority groups, more research should be conducted to help strengthen the proposed risk stratification model to help identify and treat at-risk minority patients for medication non-adherence. In terms of implications for clinical practice, patients with all three factors have the highest risk for non-adherence; therefore, they should be the priority group for education on blood pressure control and complications, such as stroke.

\section{Acknowledgment}

The authors gratefully acknowledge the Chinese immigrant participants who took part in this study, and to the health care providers who assisted with recruitment. This study was supported in part by the following sources: John A Hartford Claire M. Fagin Fellowship; Sigma Theta Tau Alpha Chapter, University of California, San Francisco; and Sigma Theta Tau International Small Grant. The authors also wish to acknowledge Miss Ariel Wang for her assistance with data analysis and manuscript preparation.

\section{References}

Abraído-Lanza, A. F., Armbrister, A. N., Flórez, K. R., \& Aguirre, A. N. (2006). Toward a theory-driven model of acculturation in public health research.
American Journal of Public Health, 96, 13421346. doi:10.2105/ajph.2005.064980

Afifi, A. A., \& Clark, V. (1997). Computer-aided multivariate analysis (3rd ed.). New York, NY: Chapman \& Hall/CRC.

Centers for Disease Control and Prevention. (1998). Behavioral Risk Factor Surveillance System Survey Questionnaire. Atlanta, Georgia: U.S. Department of Health and Human Services, Centers for Disease Control and Prevention.

Chen, M. L., \& Hu, J. (2014). Health disparities in Chinese Americans with hypertension: A review. International Journal of Nursing Sciences, 1, 318322. doi:10.1016/j.ijnss.2014.07.002

Chen, S. L., Tsai, J. C., \& Lee, W. L. (2009). The impact of illness perception on adherence to therapeutic regimens of patients with hypertension in Taiwan. Journal of Clinical Nursing, 18, 2234-2244. doi:10.1111/j.1365-2702.2008.02706.x

Chen, X, \& Swartzman, LC. (2001). Health beliefs and experiences in Asian cultures. San Diego, CA: Academic Press.

Chockalingam, A. (2008). World Hypertension Day and global awareness. Canadian Journal of Cardiology, 24, 441-444. doi:10.1016/S0828282X(08)70617-2

Dragomir, A., Côté, R., Roy, L., Blais, L., Lalonde, L., Bérard, A., \& Perreault, S. (2010). Impact of adherence to antihypertensive agents on clinical outcomes and hospitalization costs. Medical Care, 48, 418-425. doi:10.1097/MLR. 0b013e3181d567bd

Egan, B. M., Zhao, Y., \& Axon, R. N. (2010). US trends in prevalence, awareness, treatment, and control of hypertension, 1988-2008. Journal of the American Medical Association, 303, 2043-2050. doi:10.1001/jama.2010.650

Hsu, Y. H., Mao, C. L., \& Wey, M. (2010). Antihypertensive medication adherence among elderly Chinese Americans. Journal of Transcultural Nursing, 21, 297-305. doi:10.1177/1043659609360707

Ingersoll, K. S., \& Cohen, J. (2008). The impact of medication regimen factors on adherence to chronic treatment: A review of literature. Journal of Behavioral Medicine, 31, 213-224. doi:10.1007/s10865-007-9147-y

Joint National Committee. (2003). The seventh report of the Joint National Committee on prevention, detection, evaluation, and treatment of high blood pressure (NIH Publication No. 03-5233). Bethesda, MC: National Institutes of Health.

Kramer, H., Han, C., Post, W., Goff, D., Diez-Roux, A., Cooper, R., .. . Shea, S. (2004). Racial/ethnic differences in hypertension and hypertension treatment and control in the multi-ethnic study of atherosclerosis (MESA). American Journal of Hypertension, 17, 963-970. doi:10.1016/ j.amjhyper.2004.06.001 
Li, W. W., Kuo, C. T., Hwang, S. L., \& Hsu, H. T. (2012). Factors related to medication non-adherence for patients with hypertension in Taiwan. Journal of Clinical Nursing, 21, 1816-1824. doi:10.1111/ j.1365-2702.2012.04088.x

Li, W. W., Stewart, A. L., Stotts, N. A., \& Froelicher, E. S. (2005). Cultural factors and medication compliance in Chinese immigrants who are taking antihypertensive medications: instrument development. Journal of Nursing Measurement, 13, 231-252.

Li, W. W., Stewart, A. L., Stotts, N., \& Froelicher, E. S. (2006). Cultural factors associated with antihypertensive medication adherence in Chinese immigrants. Journal of Cardiovascular Nursing, 21, 354-362. doi:10.1891/jnum.13.3.231

Li, W. W., Stotts, N. A, \& Froelicher, E. S. (2007). Compliance with antihypertensive medication in Chinese immigrants: Cultural specific issues and theoretical application. Research and Theory for Nursing Practice: An International Journal, 21, 236-254. doi:10.1891/088971807782427967

Li, W. W., Wallhagen, M. I., \& Froelicher, E. S. (2008). Hypertension control, predictors for medication adherence and gender differences in older Chinese immigrants. Journal of Advanced Nursing, 61, 326-335. doi:10.1111/j.1365-2648.2007.04537.x

Long, S. J., \& Freese, J. (2006). Regression models for categorical dependent variables using Stata (2nd ed.). College Station, TX: Stata Press.
Morisky, D. E., Green, L. W., \& Levine, D. M. (1986). Concurrent and predictive validity of a selfreported measure of medication adherence. Medical Care, 24, 67-74. doi:10.1097/00005650198601000-00007

Sheng, C. S., Liu, M., Kang, Y. Y., Wei, F. F., Zhang, L., Li, G. L., . . . Wang, J. G. (2013). Prevalence, awareness, treatment and control of hypertension in elderly Chinese. Hypertension Research, 36, 824-828. doi:10.1038/hr.2013.57

Turner, B. J., Hollenbeak C., Weiner, M. G., Ten Have, T., Roberts, C. (2009). Barriers to adherence and hypertension control in a racially diverse representative sample of elderly primary care patients, Pharmacoepidemiology Drug Safety, 18, 672-81. doi: $10.1002 /$ pds. 1

Taiwan Government Information Office, Republic Of China. (2015). Chinese culture. Retrieved from http://www.taiwan.gov.tw/ct.asp?xItem=140098 $\&$ CtNode $=3837 \& \mathrm{mp}=1$

U.S. Census Bureau. (2012, March). The Asian population: 2010 Census Brief. Allegany County, NY: U.S. Census Bureau.

U.S. Census Bureau. (2013). 2013 American Community Survey. American FactFinder. Allegany County, NY: U.S. Census Bureau. Retrieved from https://www.census.gov/programssurveys/acs/data/summary-file.2013.html

World Health Organization. (2016). Raised blood pressure: Situation and trends. Retrieved from http://www.who.int/gho/ncd/risk_factors/blood_p ressure_prevalence_text/en/ 